\title{
O programa de assistência pré-natal nos Cuidados de Saúde Primários em Portugal - uma reflexão
}

\author{
The prenatal care program in Primary Care in Portugal - a reflection \\ El programa de vigilancia prenatal en la Atención Primaria en Portugal - una reflexión
}

\section{Maria de Fátima da Silva Vieira Martins'}

'Universidade do Minho, Escola Superior de Enfermagem. Braga, Portugal.

Submissão: 08-01-2013 Aprovação: 17-09-2014

\section{RESUMO}

A melhoria da qualidade dos cuidados prestados à grávida e ao recém-nascido é uma das áreas de intervenção prioritária do Plano Nacional de Saúde. Embora se reconheça que as medidas introduzidas nos últimos anos têm contribuído para diminuir os valores da mortalidade materna e perinatal, é necessário referir, também, que continuam a ocorrer gravidezes não planeadas que, não raras vezes, resultam do início tardio, ou mesmo da ausência, da assistência pré-natal. Neste artigo, procuramos refletir sobre a assistência pré-natal no contexto de saúde reprodutiva, de forma a constituir um contributo para os enfermeiros que prestam uma assistência integral e humanizada às grávidas e às suas famílias. Concluímos que a assistência pré-natal engloba um conjunto de cuidados específicos dirigidos a um grupo vulnerável, constituindo uma área muito importante na avaliação dos cuidados de saúde primários.

Descritores: Saúde Sexual e Reprodutiva; Pré-Natal; Educação em Saúde; Humanização da Assistência.

\begin{abstract}
The quality improvement of care provided to the pregnant women and newborn is one of the priority areas for intervention of the National Health Plan. While acknowledging that the measures introduced in recent years have contributed to lower the values of maternal and perinatal mortality, it should also be mentioned that unplanned pregnancies continue to occur, and that they often result in a delayed or absent prenatal surveillance. In this paper, we seek to reflect on the prenatal surveillance program under Primary Health Care relating to quality of health care provided in the context of reproductive health. We concluded that prenatal surveillance includes a set of specific care services targeted at a vulnerable group, constituting an important and susceptible area of evaluation in primary care.
\end{abstract}

Key words: Sexual and Reproductive Health; Prenatal Care; Health Education; Humanized Assistance.

\section{RESUMEN}

Mejorar la calidad de la atención a embarazada y recién nacido es una de las áreas prioritarias de intervención del plan nacional de salud. Aunque se reconoce que las medidas adoptadas en los últimos años han contribuido a reducir los valores de la mortalidad materna y perinatal, es necesario mencionar, también, que embarazos no planificados siguen produciéndose a menudo resultado de la aparición, o incluso ausencia, de vigilancia prenatal. En este artículo, reflexionamos sobre el programa de vigilancia prenatal en el marco de la atención primaria de salud, vinculándola con la calidad de la atención de la salud en el contexto de la salud reproductiva. Concluimos que la vigilancia prenatal comprende un conjunto de cuidados específicos dirigidos a un grupo vulnerable, lo que constituye un área sensible y evaluación importante en atención primaria.

Palabras clave: Salud Sexual y Reproductiva; Vigilancia Prenatal; Educación para la Salud; Asistencia Humanizada. 


\section{INTRODUÇÃO}

As questões relativas à Saúde Reprodutiva têm despertado o interesse de pesquisadores, de gestores e da própria sociedade por se tratar de um tema relevante para o delineamento de políticas populacionais e para o desenvolvimento socioeconómico $^{(1)}$. O controle da mortalidade infantil e o aumento da esperança de vida estimularam os Governos a criarem políticas de saúde com o objetivo de apoiarem as consultas pré-natais no âmbito da Saúde Reprodutiva. Em Portugal, verificamos que os serviços de saúde têm sido organizados no sentido de garantir uma assistência integral e humanizada às grávidas e às suas famílias. Deste modo, a assistência pré-natal tem sido considerada prioridade no desenvolvimento de programas, sendo ainda alvo de implementação de diversas atividades e experiências. A melhoria acentuada dos principais indicadores sanitários, como, por exemplo, a Taxa de Mortalidade Infantil, a Taxa de Mortalidade Materna e a Percentagem de Partos realizados com assistência, são dados importantes que demonstram a eficácia das políticas portuguesas implementadas ao longo dos tempos. Este artigo procura refletir sobre a assistência pré-natal no contexto de saúde reprodutiva, de forma a constituir um contributo para os enfermeiros que prestam uma assistência integral e humanizada às grávidas e às suas famílias.

\section{ASSISTÊNCIA PRÉ-NATAL NO CONTEXTO DA SAÚDE REPRODUTIVA}

A Saúde Reprodutiva é uma das áreas de cuidados de saúde consideradas prioritárias no quadro das Estratégias de Saúde aprovadas pelos mais diversos Governos de Portugal e integra os cuidados pré-concecionais, pré-natais, de planejamento familiar e de puerpério, bem como o direito à saúde sexual, como, aliás, se pode constatar da Carta dos Direitos Reprodutivos. Com a implementação destas políticas pretende-se, entre outros, fomentar as relações de dignidade, de equidade e de respeito entre sexos.

O conceito de Saúde Reprodutiva foi abordado, pela primeira vez, em 1994, na Conferência Internacional sobre a População e Desenvolvimento que teve lugar no Cairo, sendo percebido como um estado de bem-estar físico, psíquico e social e não apenas a mera ausência de doença ou enfermidade, em tudo o que diz respeito ao sistema reprodutivo, bem como às suas funções e processos ${ }^{(2)}$. Foi a partir desta Conferência que este conceito foi plenamente aceito e difundido. Este conceito requer que as pessoas possam ter uma vida sexual satisfatória e segura e a capacidade de se reproduzir e decidir se, quando e com que frequência o fazem. Neste âmbito, é importante garantir o direito à informação e ao acesso a métodos de planejamento familiar da sua escolha, que sejam seguros, eficazes e aceitáveis e, ainda, o acesso a serviços de saúde adequados, que permitam às mulheres vivenciarem uma gravidez e um parto em segurança e ofereçam aos casais as melhores oportunidades de terem crianças saudáveis. Compreende, também, o direito à saúde sexual, entendida como potenciadora da vida e das relações interpessoais ${ }^{(2)}$.
Os cuidados a prestar em Saúde Sexual e Reprodutiva constituem, deste modo, um conjunto diversificado de serviços, técnicas e métodos que visam a saúde e o bem-estar reprodutivos de mulheres e homens ao longo do seu ciclo de vida. Podemos vislumbrar da própria definição que este não é apenas um conceito médico ou biológico, incluindo nele aspetos sociais e culturais. A promoção da saúde sexual e reprodutiva dos indivíduos é um importante contributo para a formação pessoal e social.

Ao longo da vida da mulher, surgem momentos especialmente vulneráveis associados não só à sua saúde reprodutiva mas, também, a fenómenos relacionados com o papel social que a mesma desempenha ${ }^{(3)}$. O nascimento de uma criança é um acontecimento primordial na vida das famílias, pelo que a saúde da mulher durante a gravidez é um bem considerado indispensável. A mudança do contexto histórico social ao longo do século passado contribuiu, claramente, para a evolução da Saúde Reprodutiva em Portugal. Durante séculos, os cuidados prestados às mães e às crianças eram considerados assuntos domésticos, sob o foro exclusivo das mães e das parteiras. Todavia, com o decorrer dos tempos, o nível de responsabilidade foi-se alargando, tendo-se transformado, gradualmente, numa prioridade de saúde pública ${ }^{(4)}$. A prestação de cuidados de saúde, tal como a vemos hoje, organizada e com recursos físicos e humanos adequados, exercida por profissionais devidamente formados, é extremamente recente ${ }^{(5)}$. Assim, nos dias de hoje, a saúde da mulher e da criança constituiu uma prioridade da política de saúde portuguesa cujo objetivo fundamental é a melhoria da prestação de cuidados deste grupo populacional. Deste modo, é importante enquadrar a assistência pré-natal num projeto político que responda às necessidades e às exigências da sociedade, zelando pela saúde dos seus cidadãos e garantindo o acesso aos cuidados ${ }^{(6)}$.

Na Europa, designadamente na França, na Dinamarca ou na Bélgica, iniciou-se há já sessenta anos, a formalização dos sistemas de saúde para a grávida, assentes em consultas de rotina com intervalos regulares ${ }^{(7)}$, muito embora, ao longo deste tempo, tenha havido uma evolução no número de consultas recomendadas. Na verdade, não existe ainda consenso no que diz respeito ao número ideal de consultas durante a gravidez. A maioria dos autores sugere a seguinte periodicidade: uma consulta de 4 em 4 semanas, ou seja, mensal até à $32^{\text {a }}$ semana; uma consulta de 2 em 2 semanas, isto é, quinzenal, da $33^{\mathrm{a}}$ à $37^{\mathrm{a}}$ semana e uma consulta semanal, a partir da $38^{\mathrm{a}}$ semana ${ }^{(8)}$. Em Portugal, segundo a Direcção-Geral da Saúde ${ }^{(9)}$, as consultas de assistência pré-natal são marcadas mensalmente até à trigésima sexta semana de gravidez, quinzenalmente até à quadragésima semana e, a partir daqui, semanalmente, se a gravidez se prolongar até à quadragésima segunda semana. No entanto, devido a condicionalismos dos centros de saúde, estas consultas podem ser marcadas em esquema reduzido, num mínimo de seis consultas. As razões apontadas para a diferenciação do número de consultas estão, sobretudo, relacionadas com a situação clínica da mulher grávida. Em nossa opinião, o número de consultas é uma variável fundamental para que os cuidados pré-natais se mostrem adequados. Importa ainda destacar aqui o momento em que se realiza a primeira consulta, 
bem como a profundidade e a qualidade do atendimento que devem estar subjacentes à mesma.

Nesta circunstância, o desafio das autoridades políticas, dos profissionais de saúde e da própria comunidade é, primordialmente, lutar para garantir às mulheres as medidas de promoção de saúde em todo o seu processo de vida e, mais especificamente, durante o ciclo gravídico-puerperal. Jacques ${ }^{(10)}$, estudando alguns aspetos relativos ao processo de nascimento, descreve o aparecimento de regras científicas consistentes para a assistência das mulheres durante a gravidez, explanando a importância das futuras mães adquirirem uma consciência do papel que vão desempenhar. A maioria dos autores considera o pré-natal como a assistência prestada à futura mãe durante a sua gestação, envolvendo o feto em desenvolvimento, identificando, diagnosticando e tratando das diferentes respostas, quer fisiológicas, quer psicossociais ou culturais da mulher e da família. De fato, é necessário realizar uma adequada avaliação dos riscos psicossociais suscetíveis de comprometer o bem-estar futuro da mulher, da criança e da própria família. Na esteira deste objetivo, os serviços de saúde têm sido reorganizados de modo a garantir uma assistência integral e humanizada às mulheres, redirecionando um modelo vigente curativo e intervencionista, no sentido de fortalecer as intervenções preventivas e de promoção de saúde ${ }^{(4)}$.

Em Portugal, as políticas de saúde, bem como as políticas sociais, atribuem uma importância capital ao nascimento e, por via disso, desenvolveram medidas específicas a favor das mulheres grávidas. Estas políticas asseguraram um mínimo de cuidados necessários durante a gravidez, cujos ganhos observados, comparativamente ao passado, quanto à diminuição da mortalidade materna e perinatal, são testemunhos inequívocos do longo caminho já trilhado. Estes não são apenas indicadores de saúde, representando, principalmente, uma melhoria das condições socioeconómicas e da qualidade dos serviços de saúde disponíveis ${ }^{(11)}$.

De fato, nos últimos trinta e cinco anos, o nosso país tem demonstrado uma extraordinária melhoria de alguns índices, tais como: a Taxa de Mortalidade Infantil e a Taxa de Mortalidade Materna, o que comprova a preocupação do Estado na produção de políticas capazes de responder às necessidades populacionais, evidenciando ainda que a saúde pública se generalizou durante este período. Importa salientar ainda que, desde 1998, Portugal deixou de ocupar o último lugar da União Europeia no que diz respeito à Taxa de Mortalidade Infantil, encontrando-se agora numa posição mais favorável do que a Irlanda, a Grécia e o Reino Unido. Quanto à Taxa de Mortalidade Materna, em 2000, cifrava-se em 2,5\%0 e os partos sem assistência eram, nesse mesmo ano, de 0,15\%. Porém, o decréscimo destas taxas não é apenas devido às políticas de saúde, relacionando-se também com a melhoria das condições de vida, com as mudanças nos comportamentos individuais, com a melhoria das condições no trabalho e, sobretudo, com um maior nível de instrução. A título de exemplo, podemos salientar uma melhor nutrição resultante de maiores aportes alimentares, de uma alteração dos hábitos alimentares no sentido de reduzir a ingestão de calorias, de gorduras, de sal ou de açúcar, associadas à prática de exercício físico moderado, aos avanços na higiene, bem como à assistência periódica de saúde ${ }^{(4)}$.

Não menos importante, destacamos a qualidade dos cuidados de saúde que conduzem à saúde e ao bem-estar da população, assim como a formação dos profissionais de saúde que intervêm no processo de nascimento. A Organização Mundial de Saúde ${ }^{(12)}$ aponta a enfermeira especialista em saúde materna e obstétrica como a profissional mais adequada e com menor custo de efetividade, para ser responsável pela assistência de saúde durante a gravidez e o parto normal. Todavia, a escassez de enfermeiros especialistas nesta área e, consequentemente, no âmbito dos Cuidados de Saúde Primários, reflete-se negativamente na qualidade dos cuidados a prestar à mulher e à família durante a gravidez.

De igual modo, pretendemos salientar algumas das estratégias referidas no Plano Nacional de Saúde, orientado por prioridades, e definidas no âmbito de programas de saúde de 2004 a 2010. Assim, a Direcção-Geral da Saúde realçou, entre outros, a necessidade de aumentar o número de casais que realiza, pelo menos, uma consulta médica durante o período pré-concecional, tendo subjacente os seguintes objetivos: preparar a gravidez, dar continuidade ao reforço das garantias em saúde sexual e reprodutiva e fortalecer a promoção de comportamentos saudáveis durante a gravidez, principalmente aqueles que visam a diminuição de risco, tais como o consumo de tabaco e de álcool e a possibilidade de contrair infeções sexualmente transmissíveis. Destaca-se ainda a necessidade de realizar a preparação para o parto, quer física quer psiquicamente, juntamente com os profissionais de saúde disponíveis ${ }^{(13)}$.

É unânime considerar-se que os cuidados de saúde prestados à grávida, ao feto, ao recém-nascido e à família, influenciam a mortalidade e a morbilidade materna e fetal. Os cuidados pré-natais eficazes e adequados, previnem o atraso de crescimento intrauterino, o parto pré-termo e a morte perinatal ${ }^{(7-8,14-15)}$. Face aos resultados alcançados, a implementação dos cuidados pré-natais afigura-se já como uma relevante história de sucesso. Contudo, os profissionais de saúde poderão atuar ainda melhor na implementação destes cuidados, promovendo intervenções mais eficazes. Na verdade, estes profissionais podem otimizar o uso dos cuidados pré-natais, ajudando as mães a preparem-se para o parto e para a maternidade, uma vez que, não obstante a evolução retratada, persiste uma enorme necessidade de informação e de instrução ${ }^{(6)}$.

Portugal ${ }^{(3)}$ relembra a necessidade da rede de Cuidados de Saúde Primários organizar serviços multiprofissionais orientados para a promoção de saúde da mulher e da sua família, designadamente, quanto ao aconselhamento, à prestação de informação ou a educação para a saúde. É neste contexto que a enfermeira especialista em saúde materna e obstétrica tem um papel fundamental no desenvolvimento destas consultas. Não podemos esquecer que o estabelecimento de uma adequada relação entre a mulher e a enfermeira, antes e durante a gravidez, encoraja a confiança e promove a comunicação nas futuras mães de forma a melhorar o sucesso da assistência pré-natal, contribuindo para que esta recorra, mais precocemente, à consulta e adira, incondicionalmente, às suas recomendações. 
Neste sentido, a Direcção-Geral da Saúde elaborou um conjunto de Orientações Técnicas com o objetivo de uniformizar e melhorar o nível de cuidados prestados durante a gravidez, tornando-se importante a avaliação dessas medidas ${ }^{(15)}$. A norma "Orientações Técnicas n 2", denominada Vigilância Pré-Natal e Revisão do Puerpério ${ }^{(9)}$, têm por finalidade fornecer aos profissionais de saúde orientações indispensáveis para conseguir um impacto positivo na qualidade da Saúde Materna e Fetal. Nestas orientações é apresentado um esquema de assistência segundo a idade gestacional:

- Da $8^{\mathrm{a}}$ à $12^{\mathrm{a}}$ semana: história clínica e fatores de risco, exame ginecológico com palpação mamária, informação alimentar e de saúde, informação sobre a necessidade do registo pela grávida da data dos primeiros movimentos fetais, pedido dos exames laboratoriais, início do preenchimento do Boletim de Saúde da Grávida (B.S.G.);

- Da $18^{\mathrm{a}}$ à $22^{\mathrm{a}}$ semana: confirmação do tempo da gestação, auscultação dos ruídos cardíacos fetais, registos dos primeiros movimentos fetais, avaliação dos exames pedidos, eventual suporte vitamínico mineral;

- Da $28^{\mathrm{a}}$ a $32^{\mathrm{a}}$ semana: continuação da assistência e do bem-estar materno fetal, evolução da tensão arterial, altura uterina, peso, movimentos fetais e ruídos cardíacos fetais, repetição das rotinas laboratoriais;

- Da $36^{\mathrm{a}}$ a $40^{\mathrm{a}}$ semana: avaliação das condições para o parto em função do grau de risco e da distância hospitalar, informação sobre contraceção e importância da consulta da revisão de puerpério.

Iniciadas, de preferência, logo após a primeira ausência de menstruação, as consultas pré-natais oferecem a oportunidade para assegurar eficazmente a saúde da mãe e do seu filho. A avaliação inicial abarca uma história de saúde completa, ostentando a história da gestação atual, das gestações anteriores, da família, uma avaliação do perfil psicossocial, a realização do exame físico e, finalmente, a investigação e avaliação dos riscos potenciais. Os objetivos da assistência consistem na obtenção de um impacto positivo na qualidade da saúde materna e fetal, avaliar o bem-estar materno e fetal através de parâmetros clínicos e laboratoriais criteriosos, detetar precocemente fatores de risco que possam afetar a evolução da gravidez e bem-estar do feto, orientar corretamente cada situação e promover a educação para a saúde, integrando o aconselhamento e o apoio psicossocial( ${ }^{(9)}$.

Em cada uma destas consultas, a grávida é desafiada a resumir os acontecimentos mais relevantes desde a última consulta sendo questionada sobre o seu bem-estar emocional e físico, sobre eventuais desconfortos ou problemas e sobre dúvidas que possa ter. Identificam-se e exploram-se ainda as necessidades pessoais e familiares de educação para a saúde. Todavia, é importante que se estabeleça, em todas as consultas, uma relação de confiança e empatia com a grávida e/ ou família para que estas sintam a necessidade e atribuam importância à frequência das consultas. Este espaço de consultas torna-se um lugar adequado para que a grávida fale dos seus receios e das suas ansiedades que, tantas vezes, afetam negativamente a normal evolução da sua gravidez.
É indispensável cumprir o número mínimo de consultas pré-natais, mas, ainda mais importante, é a sua precocidade, havendo um consenso em recomendar o início da assistência no primeiro trimestre de gravidez ${ }^{(7-9,14,16)}$.

Apesar das diversas mudanças positivas que ocorreram a nível da saúde materno-infantil, existem barreiras no âmbito da assistência que podem condicionar a sua qualidade. Algumas investigações apontam certas variáveis, exempli gratia, a idade da mãe, o status marital, o grau de instrução, a paridade associada ao nível de prestação de cuidados e à acessibilidade, bem como os custos despendidos pela população, como suscetíveis de explicar, em parte, os condicionalismos da assistência pré-natal ${ }^{(7)}$. Remoaldo ${ }^{(7)}$ identificou três barreiras passíveis de dissuadir a utilização dos cuidados de saúde pelas grávidas: as barreiras estruturais (e.g., a organização dos serviços, a eficácia dos cuidados, o tempo e os custos); as barreiras individuais, (e.g., o conhecimento, os sentimentos e os comportamentos) e, finalmente, as barreiras sociodemográficas (e.g., a idade, o estatuto socioeconómico e o grau de instrução). Do ponto de vista educativo, estas barreiras têm importantes implicações e devem ser tidas em conta no planejamento das intervenções educativas, assim como nos cuidados a prestar à grávida. Contudo, não podemos esquecer que, hoje em dia, as mulheres também têm mais facilidade em recorrer a diversos meios de autoeducação para a saúde, tais como: revistas, livros, programas televisivos, internet, família.

As atividades de comunicação e informação em saúde necessitam de ser priorizadas no percurso do pré-natal, uma vez que o intercâmbio de informações e de experiências pode ser a melhor forma de promover a compreensão do processo da gestação, do parto e do pós-parto. Por conseguinte, os encontros pré-natais tornam-se um lugar excecional de socialização dos papéis parentais porque as redes familiares de aprendizagem, já reduzidas, são pouco enaltecidas no discurso científico e, algumas etapas da vida, são medicalizadas ${ }^{(17)}$.

Nesta linha de orientação, o contexto em que se apresenta o "Programa de Vigilância de Saúde Pré-Natal", tem que favorecer uma praxis efetiva de comunicação em saúde, visto ter como prioridade a promoção da saúde e a prevenção das doenças e complicações, cujo pilar de sustentação é exatamente a educação para a saúde. Portanto, a aproximação entre as enfermeiras e os atores sociais (grávida e família) parece ser um elemento facilitador à adequada compreensão da realidade e identificação dos traços culturais e sociais da comunidade. A interligação entre os Cuidados de Saúde Primários e Hospitalares é, de igual modo, fundamental.

\section{CONSIDERAÇÕES FINAIS}

O período pré-natal requer cuidados especiais tendo em vista o desenvolvimento adequado da gravidez, a obtenção de um parto sem complicações e o nascimento de uma criança sem problemas de saúde. Este acompanhamento concretiza-se, geralmente, nos Centros de Saúde no âmbito das consultas de assistência pré-natal, salvo raras exceções, como, por exemplo, no caso de uma gravidez de risco, onde é realizado nos Hospitais. Atualmente, em Portugal, é no âmbito dos 
Cuidados de Saúde Primários que a assistência pré-natal se desenvolve, sob a orientação específica do Médico de Família e da Enfermeira de Cuidados Gerais ou da Especialista em Saúde Materna e Obstétrica, quando esta faça parte dos quadros dos respetivos Centros de Saúde.

A reflexão efetuada permitiu salientar que a assistência de saúde assume-se como um modo tecnológico de intervenção em saúde que tende a incorporar modelos assistenciais em vigor e a articular-se com as propostas atuais de promoção de saúde. Massé(18) realça que, para além da luta contra a doença, a saúde pública propõe um novo objetivo com o intuito de mobilizar os atores em volta da gestão racional dos riscos, elaborando estruturas de intervenção responsáveis da aplicação dos programas de prevenção. Mais dans la mesure où cette "nouvelle santé publique» définit les comportements et les habitudes de vie qui sont prescrits ou proscrits, la réussite de sa mission dépendra largement de l'acceptation par la population de ce nouveau cadre normatif. Todavia, ao contemplar as necessidades sociais e determinantes socioculturais, a assistência pré-natal poderá intervir progressivamente sobre todas as situações e não unicamente sobre os fatores de risco.

\section{REFERÊNCIAS}

1. Martins MF. Surveillance prénatale dans six communes du district de Braga au Portugal. La Revue Sage-Femme. 2012;11(2):144-9

2. Ministério da Saúde (PT). Saúde Reprodutiva e Planeamento Familiar [Internet]. Lisboa (PT): Programa Nacional de Saúde Reprodutiva; 2008 [acesso em 08 de janeiro de 2013]. Disponível em: http://www.spdc.pt/files/publicacoes/11230 2.pdf

3. Portugal L. Promoção da saúde e prevenção da doença. In: Ministério da Saúde, Direção Geral da Saúde. A saúde da Mulher. Lisboa (PT): DGS; 1999. p. 107-27.

4. Martins MFSV. Educação para a saúde e vigilância pré-natal: inter-confluências profissionais e familiares [tese]. Braga (PT): Universidade do Minho; 2011.

5. Santana P, Vaz A. Os serviços de saúde e as mulheres. In: Ministério da Saúde, Direção Geral da Saúde. A saúde da Mulher. Lisboa: DGS; 1999. p. 131-65.

6. Organização Mundial de Saúde. Relatório Mundial da Saúde 2005: para que todas as mães e crianças contem [Internet]. Genebra: OMS; 2005 [acesso em 08 de janeiro de 2013]. Disponível em: http://www.who.int/whr/2005/ media_centre/overview_pt.pdf

7. Remoaldo PCA. Desigualdades territoriais e sociais subjacentes à mortalidade infantil em Portugal. Lisboa (PT): Fundação Calouste Gulbenkian; 2002.

8. Graça LM, Andrade L. Medicina materno-fetal: fundamentos e prática clínica. Lisboa (PT): Wyeth Lederle; 1996.

9. Ministério da Saúde (PT). Vigilância Pré-natal e revisão de puerpério, Orientações Técnicas. Lisboa (PT): Núcleo de Saúde Materna e Planeamento Familiar, Direcção-Geral dos Cuidados de Saúde Primários; 1989.

10. Jacques B. Sociologie de I'accouchement. Paris: Presses
Universitaires de France; 2007

11. Ministério da Saúde (PT). Ganhos de saúde em Portugal: ponto de situação: relatório do Diretor Geral e Alto-Comissário da Saúde. Lisboa (PT): Direcção-Geral da Saúde; 2002.

12. Organização Mundial de Saúde. Assistência no parto normal: um guia prático [Internet]. Genebra: OMS; 1996 [acesso em 08 de janeiro de 2013]. Disponível em: http:// abenfo.redesindical.com.br/arqs/materia/56 a.pdf

13. Ministério da Saúde (PT). Plano Nacional de Saúde 20042010: mais saúde para todos [Internet]. Lisboa (PT): Direcção-Geral da Saúde; 2004 [acesso em 08 de janeiro de 2013]. Disponível em: http://www.cm-cascais.pt/sites/default/files/ anexos/gerais/planonacionaldesade_orientaesestratgicas.pdf

14. Cunningham FG, Mac Donald PC, Gant NF, Leveno KJ, Gilstrap LC, Hankins GDV, et al. Williams Obstetrícia. 20. ed. Rio de Janeiro: Guanabara Koogan; 2000. p. 164-94.

15. Cabral SR. Avaliação da qualidade do seguimento da gravidez na Unidade de Saúde de Vialonga. Rev Port Med Geral Fam [Internet]. 2003 [acesso em 08 de janeiro de 2013];19:431-40. Disponível em: file:///C:/Users/Cliente/ Downloads/9970-9888-1-PB.pdf

16. Almeida AN. Fecundidade e contraceção. Lisboa (PT): Instituto de Ciências Sociais; 2004.

17. Valentini $\mathrm{H}$. De quoi parle-t-on dans les rencontre prénatales?. In: Saillant F, O’Neill M. Accoucher Autrement. Repères historiques, sociaux et culturels de la grossesse et de l'accouchement au Québec. Montréal: Les Éditions Saint-Martin; 1987. p. 308-404.

18. Massé R. La santé publique comme projet politique et projet individuel. In: Hours B. Systèmes et politiques de santé: de la santé publique à I'anthropologie. Paris: Les Éditions Karthala; 2001. 